\title{
Poppers-Associated Retinal Toxicity
}

TO THE EDITOR: "Poppers" (slang for various forms of alkyl nitrite) are volatile nitric oxide donors that have been used for decades as recreational drugs. Both the popularity of and legal tolerance for poppers have led to the perception that these drugs are relatively innocuous. ${ }^{1}$ Here, we describe four patients who were seen within a few months of one another and who had prolonged visual loss as a result of damage to foveal photoreceptors shortly after inhaling poppers.

In January 2010, Patient 1, a 27-year-old woman, presented with an 11-day history of a reduction in bilateral vision and a "central bright dot" in both eyes. The night before the onset of symptoms, she had attended a party, at which both she and Patient 2 had inhaled poppers (brand name, Jungle Juice) and consumed approximately half a bottle of high-grade alcohol. Patient 1 was an occasional consumer of other brands of poppers but reported having had no previous visual symptoms. Her medical history was unremarkable.

The visual acuity was $20 / 50$ in the right eye and 20/40 in the left eye. Anterior segments and intraocular pressure were normal. The fundus examination showed a yellow foveal dot in both eyes (Fig. $1 \mathrm{~A}$ and $1 \mathrm{~B}$, top row). Findings on highresolution optical coherence tomography were consistent with damage to the photoreceptor outer segment in the fovea of both eyes (Fig. 1A and $1 \mathrm{~B}$, bottom row). Color vision and full-field electroretinograms were normal. Isopropyl nitrite was identified by gas chromatography-mass spectrometry in the vapors from the popper vial. On follow-up examination 1 month later, the visual symptoms and ophthalmologic examination were unchanged.

Within a 3-month period, we examined three other patients who also had visual loss with central phosphenes after inhaling isopropyl nitrite (see the Supplementary Appendix, available with the full text of this letter at NEJM.org). Two of these patients showed resolution of symptoms over several weeks.

To our knowledge, over the past 10 years, there have been only two case reports of visual 


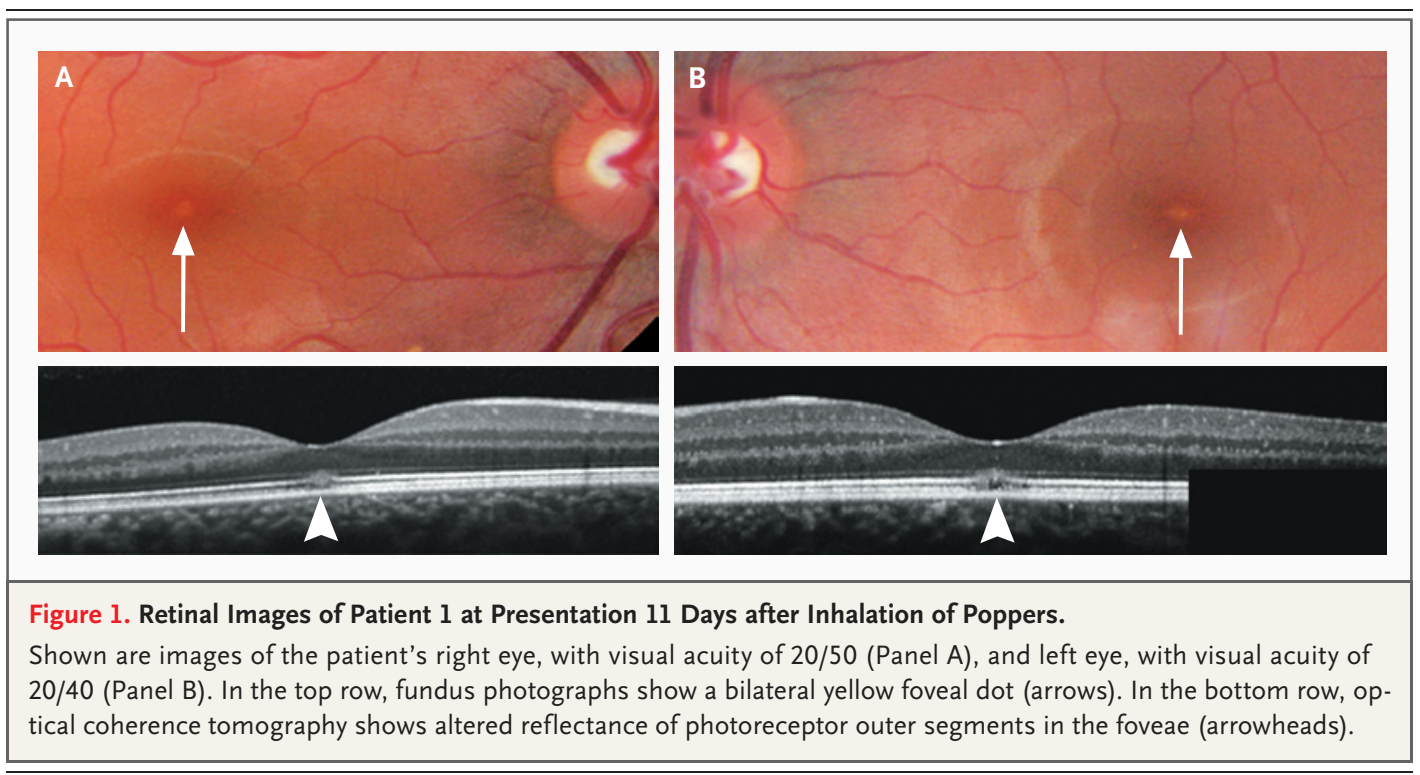

loss after inhalation of poppers, ${ }^{2,3}$ and the anatomical basis of this injury remains elusive. Thus, vision loss after intake of poppers is considered to be a rare event, although poppers-induced phosphenes are reported in Internet forums. The reason for the apparent outbreak of popper toxicity that we report here remains to be determined. It may have been due to an increased use of poppers in the population, to the availability of more powerful popper brands, or to improvements in retinal imaging technologies.

Because the only compound we detected in the vapors from the vials was isopropyl nitrite, a very potent nitric oxide donor, it is likely that the visual symptoms were due to acute, massive release of nitric oxide. Nitric oxide modulates photoreceptor metabolism and function. It also activates guanylate cyclase, ${ }^{4}$ a key enzyme for recovery of photoreceptor function after phototransduction. However, guanylate cyclase activation is predicted to decrease light sensitivity, whereas the phosphenes reported by our patients suggest increased light sensitivity. Retinal damage was functionally and anatomically limited to the foveal center, a situation similar to that observed in photic injury. Experimentally, nitric oxide is indeed known to contribute to photic injury. ${ }^{5}$ However, our patients reported that they had not stared at bright lights. Because nitric oxide is a potent vasodilator, acute changes in ocular perfusion pressure might have contribut- ed to retinal damage, although photoreceptors are relatively resistant to short-term circulatory changes.

Consumers and ophthalmologists should be aware of the possible retinal toxicity of poppers. In patients with recent bilateral visual loss and phosphenes, this diagnosis should be considered. Finally, the determination of the molecular basis for the toxic effects of poppers may be of interest to further document the role of nitric oxide in retinal function and diseases and to identify potential approaches to counter such toxicity.

\section{Catherine Vignal-Clermont, M.D.}

Fondation Ophtalmologique Adolphe de Rothschild Paris, France

Isabelle Audo, M.D., Ph.D.

José-Alain Sahel, M.D.

Michel Paques, M.D., Ph.D.

Quinze-Vingts Hospital

Paris, France

mp@cicoph.org

Supported by the Institut National de la Santé et de la Recherche Médicale, the Direction de l'Hospitalisation et des Soins, and the Foundation Fighting Blindness.

Disclosure forms provided by the authors are available with the full text of this letter at NEJM.org.

1. Romanelli F, Smith KM, Thornton AC, Pomeroy C. Poppers: epidemiology and clinical management of inhaled nitrite abuse. Pharmacotherapy 2004;24:69-78.

2. Fledelius HC. Irreversible blindness after amyl nitrite inhalation. Acta Ophthalmol Scand 1999;77:719-21.

3. Pece A, Patelli F, Milani P, Pierro L. Transient visual loss 
after amyl isobutyl nitrite abuse. Semin Ophthalmol 2004;19: 105-6.

4. Goldstein IM, Ostwald P, Roth S. Nitric oxide: a review of its role in retinal function and disease. Vision Res 1996;36:2979-94.

5. Goureau O, Jeanny JC, Becquet F, Hartmann MP, Courtois Y. Protection against light-induced retinal degeneration by an inhibitor of NO synthase. Neuroreport 1993;13:233-6.

Correspondence Copyright (๑) 2010 Massachusetts Medical Society. 